\title{
THE INVARIANT OF CHEN-NAGANO ON FLAG MANIFOLDS
}

\author{
CRISTIÁN U. SÁNCHEZ
}

(Communicated by Jonathan M. Rosenberg)

\begin{abstract}
In this paper an extension of the 2-number $\left(\#_{2}(M)\right)$ of a symmetric space is given for $k$-symmetric spaces. The new invariant is computed for flag manifolds which are not symmetric. It turns out to be equal to the EulerPoincaré characteristic.
\end{abstract}

\section{INTRODUCTION}

In a recent paper [ChN], Chen and Nagano introduced a new numerical invariant associated to compact symmetric spaces which they call the "2-number". For a compact connected symmetric space $M$ this 2 -number, $\#_{2} M$, is defined as the maximal possible cardinality of the subsets $A \subset M$ with the following property. For each $x \in A$ the symmetry $s_{x}$ of the symmetric space at the point $x$ fixes every point of $A$. This definition is equivalent to saying that $\#_{2} M$ is the maximal possible cardinality of the subsets $A \subset M$ with the property that for each pair of points $x, y$ in $A$ there exists a closed geodesic in $M$ on which $x$ and $y$ are antipodal to each other. With this last form of defining the 2-number this invariant makes sense for arbitrary connected Riemannian manifolds, but it is clear that in this general situation it is very difficult to compute this number.

In the case of symmetric spaces, however, Chen and Nagano are able to compute $\#_{2} M$ for a great deal of spaces and at the same time obtain many interesting connections between the 2-number and topological invariants of the manifold. The results of greater interest to us, which motivate the present paper, are those relating the 2-number of a compact symmetric space to its Euler characteristic $\chi(M)$. In (4.1) and (4.3) of [ChN] they prove

1.1. Theorem (Chen-Nagano). (i) For a compact connected symmetric space $M, \chi(M) \leq \#_{2} M$.

(ii) If $M$ is a compact hermitian symmetric space of semisimple type, then $\chi(M)=\#_{2} M$.

Now if one wants to extend these results to more general homogeneous spaces, there are two ways of doing this. One is to consider general compact homogeneous Riemannian spaces where one may try to study $\#_{2} M$, and the other one

Received by the editors December 19, 1991.

1991 Mathematics Subject Classification. Primary 53C30; Secondary 53C35.

Key words and phrases. 2-Number, $k$-number, $k$-symmetric spaces, flag manifolds.

This research was supported by a grant from the Consejo de Investigaciones Cientificas y Técnicas de la Provincia de Córdoba (CONICOR), Argentina. 
is to extend the definition of the 2-number of a " $k$-number" for $k$-symmetric spaces (regular $s$-manifolds of order $k$ in the sense of [K]), of which ordinary symmetric spaces are a particular case. From our point of view this last procedure gives coherence to the whole picture of $k$-symmetric spaces, and at the same time it is a genuine extension of the problem. As it is well known, there are many conspicuous examples of $k$-symmetric spaces which are not 2-symmetric. They are interesting objects of study, and it seems worthwhile to obtain geometric information about their topological invariants. On the other hand, it is clear that the study of $\#_{2} M$ in Riemannian homogeneous spaces seems just as difficult as for general Riemannian manifolds.

\section{THE $k$-NUMBER}

Let us recall the definition of $k$-symmetric space. If $M$ is a connected Riemannian manifold, an isometry of $M$ with isolated fixed point $x$ is called a symmetry of $M$ at $x$. A family $\left\{\theta_{x}: x \in M\right\}$ of symmetries is called an $s$-structure on $M$. The $s$-structure is said to be of order $k$ if $\theta_{x}^{k}=\operatorname{id}(M)$ for each $x \in M$ and $\theta_{x}^{r} \neq \operatorname{id}(M)$ for all $r<k$. Furthermore, if the $s$-structure satisfies

$$
\theta_{x} \circ \theta_{y}=\theta_{z} \circ \theta_{x}, \quad \text { where } z=\theta_{x}(y),
$$

then $M$ is said to be a regular $s$-manifold. If $M$ has an $s$-structure of order 2 then this structure is automatically regular and $M$ is a symmetric space in the usual sense.

Let $M$ be a compact connected Riemannian manifold with a regular $s$ structure of order $k \geq 2$. We define now the " $k$-number" $\#_{k}(M)$ as the maximal possible cardinality of the subsets $A_{k} \subset M$ with the property that for each $x \in A_{k}$ the symmetry $\theta_{x}$ fixes every point of $A_{k}$. Clearly, if $k=2$ then this is just the 2-number of $M$ defined on [ChN]. Notice that $\#_{k}(M)$ is finite.

Let $A \subset M$ be a subset such that for each $x \in A$ the symmetry $\theta_{x}$ fixes every point of $A$, and assume that the cardinality of $A$ is precisely $\#_{k}(M)$. We shall say that this subset $A$ is a \#-set of $M$. Let $a \in A$; since $M$ is homogeneous, we may take it as the base point. By definition, $A$ is contained in the fixed point set of the symmetry which we shall denote by $F\left(\theta_{a}, M\right)$. Since $a$ is an isolated fixed point of $\theta_{a}$, we have $F\left(\theta_{a}, M\right)=\{a\} \cup N_{1} \cup N_{2} \cup \cdots \cup N_{j}$ where each $N_{s}$ is a connected totally geodesic submanifold of $M$, which is itself a $k$-symmetric space. Now let $A_{s}=A \cap N_{s}$. Then we clearly have that the cardinality of $A_{s}$, \# $A_{s}$, satisfies $\# A_{s} \leq \#_{k}\left(N_{s}\right)$, and therefore we immediately have the following formula, which was established for $k=2$ in [ChN],

$$
\#_{k}(M)-1 \leq \sum_{i=1}^{j} \#_{k}\left(N_{i}\right) .
$$

Given a compact manifold $M$ we shall denote by $\beta_{i}\left(M, Z_{p}\right)$ the $i$ th Betti number of $M$ with coefficients in $Z_{p}$ and put $b\left(M, Z_{p}\right)=\sum_{i} \beta_{i}\left(M, Z_{p}\right)$.

(2.3) Proposition. Let $M$ be a p-symmetric manifold with $p \geq 2$ a prime number. Then

$$
\#_{p}(M) \leq b\left(M, Z_{p}\right)
$$


Proof. Let us proceed by induction on the dimension of $M$. The statement is obviously true if this dimension is zero. Let us assume that the proposition is true for every $p$-symmetric space $N$ such that $\operatorname{dim} N=k<n$, and take now our $n$-dimensional manifold $M$. Let $A \subset M$ be a subset such that $\# A=$ $\#_{p}(M)$, and take $a \in A$. Then as before, $A \subset F\left(\theta_{a}, M\right)=\{a\} \cup N_{1} \cup \cdots \cup N_{j}$, and by $(2.2)$ and the inductive hypothesis we have

$$
\#_{p}(M)-1 \leq \sum_{i=1}^{j} \#_{p}\left(N_{i}\right) \leq \sum_{i=1}^{j} b\left(N_{i}, Z_{p}\right) .
$$

Now by considering the subgroup of the isometry group of $M$ generated by $\theta_{a}$, we have an action of $Z_{p}$ on $M$, and by [B, p. 144, 7.9] we know the inequality

$$
\sum_{i \geq s} \beta_{i}\left(F\left(\theta_{a}, M\right), Z_{p}\right) \leq \sum_{i \geq s} \beta_{i}\left(M, Z_{p}\right),
$$

which in particular implies

$$
b\left(F\left(\theta_{a}, M\right), Z_{p}\right) \leq b\left(M, Z_{p}\right) .
$$

It follows now from (2.4) that

$$
\#_{p}(M) \leq 1+\sum_{i=1}^{j} b\left(N_{i}, Z_{p}\right)
$$

and since $b\left(\{a\}, Z_{p}\right)=1$, we obtain

$$
\#_{p}(M) \leq b\left(F\left(\theta_{a}, M\right), Z_{p}\right) \leq b\left(M, Z_{p}\right),
$$

which proves the proposition.

Proposition (2.3) can be extended to the case in which the order of the $k$ symmetric space is not prime.

(2.6) Proposition. Let $M$ be a compact k-symmetric space and $p$ a prime number which divides $k$. Then

$$
\#_{k}(M) \leq b\left(M, Z_{p}\right) \text {. }
$$

Proof. Let $k=t p$, and choose, as in the proof of (2.3), the subset $A \subset M$ such that $\# A=\#_{k}(M)$. Take $a \in A$ and consider the isometry of $M$ defined by $\delta_{a}=\theta_{a}^{t}$. Clearly $\delta_{a}^{p}=\mathrm{id}(M)$ and again by considering the subgroup of the group of isometries generated by $\delta_{a}$ we obtain an action of $Z_{p}$ on $M$. Now $F\left(\delta_{a}, M\right)=N_{1} \cup \cdots \cup N_{h}$ where $a \in N_{1}$ and each $N_{i}$ is a connected component of the fixed point set. Each of these submanifolds is easily seen to be a $k$-symmetric space; the manifold $N_{\mathrm{l}}$ is, in fact, $t$-symmetric, but this fact will not be used in the present paper.

The proof will now continue by induction on the dimension of the manifold $M$. It is obvious that the proposition is true if $\operatorname{dim} M=0$. Let us assume then that the statement is true for each $k$-symmetric space $N$ such that $\operatorname{dim} N=$ $r<n$, and consider our $n$-dimensional manifold $M$ and the subset $A \subset M$ as above. Clearly $A \subset F\left(\delta_{a}, M\right)$ and then

$$
\#_{k}(M)=\# A=\sum_{i=1}^{h} \#\left(A \cap N_{i}\right) \leq \sum_{i=1}^{h} \#_{k}\left(N_{i}\right) .
$$


Now by the induction hypothesis we have

$$
\#_{k}(M) \leq \sum_{i=1}^{h} b\left(N_{i}, Z_{p}\right)=b\left(F\left(\delta_{a}, M\right), Z_{p}\right),
$$

and since $M$ is again supporting an action of $Z_{p}$, by (2.5) we have

$$
b\left(F\left(\delta_{a}, M\right), Z_{p}\right) \leq b\left(M, Z_{p}\right),
$$

which completes the proof of the proposition.

(2.7) Corollary. Let $M$ be a compact $k$-symmetric space, and let $\left\{p_{i}: 1 \leq\right.$ $i \leq s\}$ be the set of all the prime numbers which divide $k$. Then $\#_{k}(M) \leq$ $\operatorname{Min}\left\{b\left(M, Z_{p_{i}}\right): 1 \leq i \leq s\right\}$.

\section{THE $k$-NUMBER FOR EXTRINSIC $k$-SYMMETRIC MANIFOLDS}

In this section we give a condition under which we obtain a converse of the results of the previous one.

Let $M$ be a compact connected $n$-dimensional Riemannian manifold, and let $i: M \rightarrow R^{n+q}$ be an isometric embedding which has the following properties.

(i) For each $x \in M$ there is an isometry $\sigma_{x}: R^{n+q} \rightarrow R^{n+q}$ such that $\sigma_{x}^{k}=$ identity, $\sigma_{x}(x)=x$, and $\sigma_{x} \mid M_{x}^{\perp}=$ identity on $M_{x}^{\perp}$, where $M_{x}^{\perp}$ denotes the normal space to $M$ at $x$.

(ii) $\sigma_{x}(M) \subseteq M$.

(iii) Let $\theta_{x}=\left(\sigma_{x} \mid M\right)$. The collection $\left\{\theta_{x}: x \in M\right\}$ defines on $M$ a Riemannian regular $s$-structure of order $k$ [K, pp. 4-6], as was defined in $\S 2$.

If conditions (i), (ii), and (iii) are satisfied, we say that $M$ is an extrinsic $k$-symmetric submanifold of $R^{n+q}$.

To mention examples of these submanifolds we indicate that the spheres of dimensions 6, 5, and 2 are examples of orders $k=3,4$ and arbitrary [S1]. Every generalized flag manifold has an embedding with this property [S2].

(3.1) Theorem. Let $M^{n} \subset R^{n+q}$ be an extrinsic $k$-symmetric submanifold. If $p \geq 2$ is a prime number which divides $k$ then

$$
\#_{k}(M)=b\left(M, Z_{p}\right) \text {. }
$$

Proof. Let $V=R^{n+q}$ and $S(V)=\{v \in V:\|v\|=1\}$. For each $v \in S(V)$ let $h_{v}$ denote the "height" function in the direction of $v$, i.e., $h_{v}(x)=\langle v, x\rangle \forall x \in V$. The following basic principle is well known and frequently used in the literature; we know no reference for it, but it seems to be a "folk theorem".

For almost all $v \in S(V)$ the function $h_{v}$ is stable in $M$; that is, $h_{v}$ is a Morse function with only one critical point in each critical level.

For each critical point $x$ of $h_{v}$ (a stable height function), the isometry $\sigma_{x}$ leaves $h_{v}$ invariant since $v$ is normal to $T_{x}(M)$, the tangent space to $M$ at $x$. Now

$$
h_{v}\left(\sigma_{x}(y)\right)=\left\langle v, \sigma_{x}(y)\right\rangle=\left\langle\sigma_{x}(v), \sigma_{x}(y)\right\rangle=h_{v}(y) \quad \forall y \in M .
$$

Then, for fixed $h_{v}$ and $x$, the critical point of $h_{v}$, we have that $\sigma_{x}$ fixes every critical point of $h_{v}$. In fact, if $a$ is a critical point of $h_{v}$, let $s=\sigma_{x}(a)$. Then $s$ is another critical point of $h_{v}$. This is so because if $Y \in T_{s}(M)$ then $Y=\left.\sigma_{x *}\right|_{a} X$ for some $X \in T_{a}(M)$ and, therefore,

$$
\left.d h_{v}\right|_{s} Y=\left.\left.d h_{v}\right|_{s} \sigma_{x *}\right|_{a} X=\left.d\left(h_{v} \circ \sigma_{x}\right)\right|_{a} X=\left.d h_{v}\right|_{a} X=0 ;
$$


but since (3.2) implies $h_{v}(s)=h_{v}(a)$ and $h_{v}$ has only one critical point on each critical level, we have $s=a$ and so $a$ is a fixed point of $\sigma_{x}$.

Let $A_{v}$ be the set of critical points of $h_{v}$. Then this set has the property that the symmetry of $M$ at each point of $A_{v}$ fixes every other point of $A_{v}$. It follows immediately that $\# A_{v} \leq \#_{k}(M)$, and in turn, Proposition (2.6) yields

$$
\# A_{v} \leq \#_{k}(M) \leq b\left(M, Z_{p}\right) \text {. }
$$

On the other hand, if we denote by $\mu_{i}\left(h_{v}\right)$ the number of critical points of index $i$ of the function $h_{v}$ and call $\mu\left(h_{v}\right)=\sum_{i \geq 0} \mu_{i}\left(h_{v}\right)$, then by definition of the set $A_{v}$ we have

$$
\mu\left(h_{v}\right)=\# A_{v} .
$$

Now we recall the Morse inequalities

$$
\mu_{j}\left(h_{v}\right) \geq \beta_{j}(M, F)
$$

where $\beta_{j}$ indicates the $j$ th Betti number of $M$ with coefficients in the arbitrary field $F$.

It follows from (3.5) that

$$
\mu\left(h_{v}\right) \geq b\left(M, Z_{p}\right),
$$

and from this, (3.3), and (3.4) we obtain

$$
\mu\left(h_{v}\right)=\# A_{v} \leq \#_{k}(M) \leq b\left(M, Z_{p}\right) \leq \mu\left(h_{v}\right) ;
$$

therefore,

$$
\mu\left(h_{v}\right)=\#_{k}(M)=b\left(M, Z_{p}\right) .
$$

This completes the proof of the theorem.

Remark. Due to a well-known result of Ferus [F], the collection of symmetric $R$-spaces coincides with that of 2-symmetric submanifolds of Euclidean spaces. Then Theorem (3.1), in the case of $k=2$, is equivalent to the main theorem of Takeuchi in [T]; therefore, our result yields a completely different proof of it. This theorem of Takeuchi is also mentioned in [ChN].

Since it is known that every generalized flag manifold admits $k$-symmetric structures, and, as we mentioned, it is extrinsic $k$-symmetric for this structure [S2], we have immediately

(3.6) Corollary. If $M$ is a generalized flag manifold then any of its $k$ symmetric structures satisfies

$$
\#_{k}(M)=\chi(M)=b(M, Z) .
$$

Clearly this result is related to Theorem (1.1). In fact, for hermitian symmetric spaces, which are generalized flag manifolds and admit a 2-symmetric structure, the corollary yields a different proof of (1.1). At the same time it places the $[\mathrm{ChN}]$ result in a general perspective.

\section{REFERENCES}

[B] G. Bredon, Introduction to compact transformation groups, Pure Appl. Math., vol. 46, Academic Press, New York and London, 1972.

[ChN] B. Y. Chen and T. Nagano, A Riemannian geometric invariant and its applications to a problem of Borel and Serre, Trans. Amer. Math. Soc. 308 (1988), 273-297. 
[F] D. Ferus, Symmetric submanifolds of Euclidean space, Math. Ann. 247 (1980), 81-93.

[K] O. Kowalski, Generalized symmetric spaces, Lecture Notes in Math., vol. 805, Springer, Berlin, Heidelberg, and New York, 1980.

[S1] C. U. Sánchez, Regular S-structures on spheres, Indiana Univ. Math J. 37 (1988), 165-180.

[S2] _ K-Symmetric submanifolds of $R^{n}$, Math. Ann. 270 (1985), 297-316.

[T] M. Takeuchi, Two-number of symmetric R-spaces, Nagoya Math. J. 115 (1989), 43-46.

Facultad de Matemtica Astronomia y Fisica, Valparaiso y R. Martinez, Ciudad UniVERsitaria, 5000 Córdoba, Argentina

E-mail address: Sánchez@uunet!smimaf.edu.ar 